\title{
Formulation and Evaluation of Hydrocortisone Sodium Succinate Mucoadhesive Buccal Tablet
}

\author{
Mohammad Ali Daneshmehr', Hilda Adibpour ${ }^{1}$, Zahra Ataie $^{2,3, *}$ \\ 'Department of Medicinal Chemistry, School of Pharmacy, Iran University of Medical Sciences, Tehran, IRAN. \\ 2Evidence-based Phytotherapy and Complementary Medicine Research Center, Alborz University of Medical Sciences, Karaj, IRAN. \\ ${ }^{3}$ Department of Pharmaceutics, Faculty of Pharmacy, Alborz University of Medical Sciences, Karaj, IRAN.
}

\begin{abstract}
Background: The present study involves the formulation and in-vitro assessment of mucoadhesive tablets of hydrocortisone sodium succinate. Local treatment for buccal inflammatory injuries such as aphthous wounds relies on the fact that a high amount of drug can be delivered at the target site, as well as reduction or avoidance of steroidal adverse effects seen by systemic administration routes. Methods: Tablets contained acacia as the mucoadhesive component with different concentrations $(10,20$ and $30 \% \mathrm{w} / \mathrm{w})$. To this, lactose, magnesium stearate and active pharmaceutical ingredient were added and mixed thoroughly. These tablets were prepared by direct compression technique. Buccal tablets were tested for thickness, hardness, weight and content uniformity, surface $\mathrm{pH}$ and mucoadhesive strength. Kinetic of drug release was also calculated for the best formulation. Dissolution parameters were also measured in-vitro for the selected formulation and compared with Corlan $®$ as the standard reference tablet. Results: All of the tests confirmed the proper formulation according to the standards of conventional oral tablets. Mucoadhesive strength of one of formulations was about $50 \%$ more than the reference
\end{abstract}

tablet. The optimized formulation that was made by $10 \%$ acacia provided adequate release comparable to Corlan ${ }^{\circledR}$. Release profile was best fitted to Hixson-Crowell model. Conclusion: We concluded that our formulated buccal tablet was comparable and similar in-vitro bioequivalent) to the reference tablets.

Key words: Aphthous, Buccal tablet, Mucoadhesive, Hixson-Crowell, In-vitro, Bioequivalence.

\section{Correspondence}

\section{Zahra Ataie}

${ }^{2}$ Evidence-based Phytotherapy and Complementary Medicine Research Center, Alborz University of Medical Sciences, Karaj, IRAN.

${ }^{3}$ Department of Pharmaceutics, Faculty of Pharmacy, Alborz University of Medical Sciences, Vali-e- Asr Blvd, Karaj, IRAN.

Phone no: 0098-2632567175

Email: zataie@abzums.ac.ir

DOI: 10.5330/ijpi.2020.3.54

\section{INTRODUCTION}

Oral buccal administration of drugs offers advantages for either local or systemic drug delivery such as ease of administration, patient acceptance, fast absorption of drug through high vascularization of buccal mucosa and avoidance from possible gastrointestinal damages. ${ }^{1}$ Previous studies showed that topical buccal therapy with corticosteroid anti-inflammatory drugs is promising in control of ulcerative and inflammatory mucosal diseases. $^{1-3}$

Several commercial products containing hydrocortisone sodium succinate and hydrocortisone acetate as a systemic and local antiinflammatory and anti-allergic agent are available for parenteral or topical use on skin. ${ }^{2}$

Mucoadhesive buccal tablets are also preferred for local delivery of active compounds because adhesion of tablets to mucosal tissue keeps them in place in direct contact to the target site like wound or lesion. Increase in residence time of medicine at the site of action in turn increases the concentration of localized drug leading to the improvement in efficiency of treatment. Besides tablets are single dosed and offer accurate dosing over other topical forms like ointments and gels. In fact by using mucoadhesive buccal tablets, high amount of steroids are released at the target site while systemic side effects are minimized or even avoided.

Developing a mucoadhesive buccal formulation for painful aphthous lesions was the aim of this study. The conventional formulation like lozenges, troches, gels and oral mouthwashes are simple dosage forms for drug delivery through the oral cavity mucosa. ${ }^{1-5}$ However, these conventional dosage forms have two major disadvantages which consist of an initial burst of activity followed by a rapid decrease in concentration and a limited in situ persistence related to the constant flow of saliva and the mobility of involved tissues. ${ }^{3,6}$ Mucoadhesive buccal preparations administered are expected to overcome above problems. ${ }^{7,8}$ Bioadhesive polymers have received considerable attention as platforms of controlled drug delivery for the following reasons. They can be localized in a specific surface, which is able to absorb drugs leading to the enhancement of bioavailability; they prolong the residence time and ensure an optimal contact with the absorbing surfaces. The mechanism of bioadhesion of polymers to the mucosa is still not fully understood. Different types of mucoadhesive polymers may be used in design of buccal drug delivery systems. Recently buccal mucoadhesive tablets have been developed with promising results. ${ }^{8-13}$

There are a number of mucoadhesive polymers that many of them are introduced recently. ${ }^{14,15}$ But acacia is a natural polymer that has been used for years. Because of its properties like being natural, safety, water solubility ${ }^{16}$ and also in order to produce a formulation comparable to Corlan tablet, acacia was selected as the mucoadhesive polymer in our study.

In this work we developed a bioadhesive tablet for buccal administration, containing hydrocortisone sodium succinate by different concentrations of mucoadhesive component acacia. ${ }^{17}$ It was then compared to the reference tablet Corlan ${ }^{\star}$ manufactured by Auden Mckenzie, North Devon, UK. The research covered formulations with varying ratios 
of mucoadhesive compound to study the effect of bioadhesive concentration on tablet characteristics. ${ }^{17-19}$

\section{MATERIALS AND METHODS}

\section{Materials}

Hydrocortisone sodium succinate was kindly gifted by Jaber ebn Hayan Pharmaceutical Co., Tehran, Iran. Lactose monohydrate, magnesium stearate and acacia were purchased from Sigma-Aldrich Co. (Germany). All other reagents and chemicals were of analytical grade.

\section{Formulation of Mucoadhesive tablet}

Different formulations were made by varying amounts of mucoadhesive component (acacia). Other ingredients included the diluent (lactose monohydrate) and the lubricant ( $1 \% \mathrm{w} / \mathrm{w}$ magnesium stearate) accompanied by our medicinal compound (hydrocortisone sodium succinate). All of ingredients, except lubricant, were homogenously blended for $20 \mathrm{~min}$, followed by further blending for $5 \mathrm{~min}$ after addition of the lubricant. Resultant mixture of powders was screened through sieve number 40 . The blend was then directly compressed using $6 \mathrm{~mm}$ flat-faced punch to $80 \mathrm{mg}$ tablet, at a constant compression load using a single punch tablet machine (Noavaran Co. Iran). Table 1 shows different formulations of our buccal tablets.

\section{Evaluation of Tablets}

Tablets were evaluated for weight variation $(n=10)$, hardness $(n=6)$, thickness $(n=6)$, friability $(n=8$, about $6.5 \mathrm{~g})$, surface $\mathrm{pH}(n=3)$, content uniformity $(n=10)$, assay $(n=5)$, Disintegration time $(n=6)$, invitro release $(n=6)$ and in-vitro bioequivalent test $(n=6)$ and in-vitro mucoadhesion study $(n=3) .{ }^{19,20}$

Weight variation test was performed for ten tablets from each batch using an electronic balance and average values were calculated. ${ }^{20}$

Hardness test was conducted for 6 tablets from each batch using hardness tester and average values were calculated.

The thicknesses of buccal tablets were determined using a micrometer. Six individual tablets from each batch were used and the average thickness was calculated. ${ }^{20}$

Friability was found to be within the limits of conventional oral tablets stated in the United States Pharmacopoeia (USP). Friability was determined by testing 8 tablets in a friability tester for $4 \mathrm{~min}$ at $25 \mathrm{rpm} .^{21,22}$ The bioadhesive buccal tablets $(n=3)$ were exposed to $1 \mathrm{ml}$ of distilled water and allowed to swell for $5 \mathrm{~min}$. The $\mathrm{pH}$ was measured after bringing the $\mathrm{pH}$ meter electrode in contact with the surface of the tablet and allowing it to equilibrate for $1 \mathrm{~min} .^{21,22}$

10 formulated tablets and 10 reference tablets were randomly selected. Hydrocortisone amount was then measured and compared to the amount found in the reference tablets according to the USP for content uniformity assessment.

Five tablets were selected randomly and powdered in a mortar. An amount of powder equivalent to the single dose of drug was dissolved in purified water by shaking for $30 \mathrm{~min}$. It was then filtered through a filter $(0.45 \mu \mathrm{m})$. The drug content was analyzed at $248 \mathrm{~nm}$ using the UV spectroscopy (CECIL 7250, UK). Each measurement was carried out in triplicate and average of drug content was calculated. ${ }^{21,22}$

For each batch, six randomly selected tablets were placed in disintegration apparatus basket and the process of disintegration was carried out. Time was recorded after complete disintegration of tablets. ${ }^{21,22}$

The USP rotating paddle method (apparatus II) was used to study the drug release from the buccal tablets. Dissolution medium was consisted of $900 \mathrm{~mL}$ purified water. Experiment was performed at $37 \pm 0.2^{\circ} \mathrm{C}$ with a rotation speed of $50 \mathrm{rpm}$. Tablets were placed at the bottom of dissolution medium and Samples $(5 \mathrm{~mL})$ were withdrawn at predetermined time intervals and replaced by an equivalent amount of fresh medium. The samples were filtered through a membrane filter $(0.45 \mu \mathrm{m})$ and analyzed by UV spectrophotometer at $248 \mathrm{~nm} .^{21,22}$ One-way ANOVA analysis was performed on the release profile to compare different formulations. $(P<0.05$, significant difference)

Amount of released hydrocortisone after $45 \mathrm{~min}$ were used as dissolution test data according to pharmacopoeia.

An in-house apparatus ${ }^{23}$ was used to quantify the mucoadhesive property of tablets in vitro (Figure 1).

The sheep buccal mucosa was cut into pieces, washed with phosphate buffer $\mathrm{pH} 6.8$ and tied onto the lower platform of the apparatus (Figure 1) with mucosal side upwards. The buccal tablet was mounted onto the upper platform of the test device that was then lowered onto the lower part until it just touched the sheep buccal mucosa. The upper platform was attached to a digital balance. A digital balance was installed above the instrument attached to the upper platform using a string. The vessel containing the platforms was filled with phosphate buffer $\mathrm{pH} 6.8$ and kept at $37 \pm 0.5^{\circ} \mathrm{C}$. Tablets were left in position in contact with mucosa for a few minutes before the application of detachment force. The lower platform was then steadily descended using the adjustable screw until detachment of tablet from the sheep tissue. Mucoadhesive strength was assessed in terms of weight (grams, g) required to detach the tablet from the membrane, which was observed by a digital balance (Figure 2). ${ }^{23,24}$

For each batch of preparation, three tablets were examined and the mean of adhesive strength was calculated. Mucoadhesive strength was measured as force of adhesion in newton by using the following formula. Force of adhesion $(\mathrm{N})=$ Mucoadhesive strength $(\mathrm{g}) \times 9.81 / 100$ FI, FII, FIII and Corlan tablets were compared using this procedure.

Table 1: Composition of different formulations of hydrocortisone buccal tablet.

\begin{tabular}{cccc}
\hline Ingredients & $\mathrm{FI}(\mathrm{mg})$ & $\mathrm{FII}(\mathrm{mg})$ & $\mathrm{FIII}(\mathrm{mg})$ \\
\hline Hydrocortisone (as sodium succinate) & 3.52 & 3.52 & 3.52 \\
Acacia & 8.0 & 16.0 & 24.0 \\
Lactose & 67.68 & 59.68 & 51.68 \\
Magnesium stearate & 0.8 & 0.8 & 0.8 \\
Total & 80.0 & 80.0 & 80.0 \\
\hline
\end{tabular}

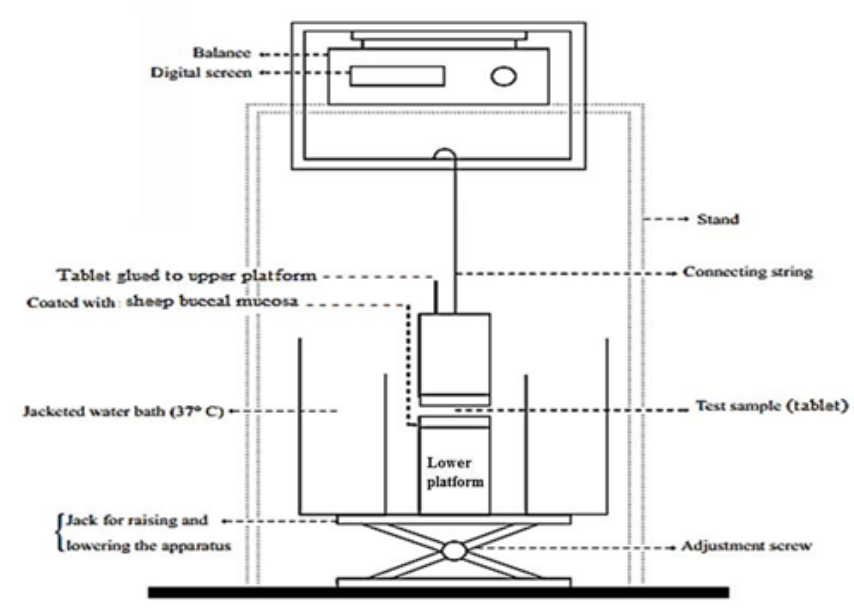

Figure 1: Diagrammatic representation of the device for mucoadhesive strength measurement. ${ }^{23}$ 
To study the release kinetic model and bioequivalence in-vitro, Corlan tablets $(n=3)$ and the best-formulated tablets $(n=3)$ were randomly selected and put inside the dissolution device. The method of study was the same as dissolution rate measurements mentioned before. Absorbance at $248 \mathrm{~nm}$ was obtained and compared to calibration curve in order to calculate the concentrations at 5, 10, 15, 30 and 45 min of experiment. Kinetic model of release was also obtained for FI formulation and Corlan tablets. Parameters of release profile for various models including zero order, first order, Higuchi, Hixson-Crowell, Weibull, linear probability, log probability, square root of mass and reciprocal powered time were calculated by using relevant formulas. The best model was selected considering the highest residual sum of squares (RSQ) and the least mean percentage of error (MPE) values. ${ }^{25}$

For bioequivalence study, difference factor (f1) and similarity factor (f2) were calculated to compare the release pattern between FI and Corlan tablets. ${ }^{26}$

\section{RESULTS}

Composition of prepared formulations is represented in Table 1. All of the performed tests were found to be within the limits of conventional oral tablets stated in the USP Results are shown in Table 2.
Calibration curve for hydrocortisone is presented in Figure 3 that was used for all hydrocortisone quantifications.

FI, FII and FIII were found to release $105.3 \%, 102.7 \%$ and $101.5 \%$ of the drug within $45 \mathrm{~min}$. The reference tablet was found to release $101.6 \%$ of the drug within 45 min. ${ }^{13,27}$

All of the hydrocortisone is therefore released in $45 \mathrm{~min}$ for our study and reference tablets. Comparison of cumulative drug release for all formulations is shown in Figure 4.

The mucoadhesive characteristics were affected by the concentration of the acacia, as any increase in concentration of acacia could increase mucoadhesive strength of formulation consequently (Table 3). The mucoadhesive strength of the reference tablet was slightly lower compared to FI, which may be related to lower diameter of reference tablet compared to our formulated tablets.

Among three concentrations of mucoadhesive component (10, 20 and $30 \%$ ), all of them had an acceptable drug release profile, mucoadhesive strength, surface $\mathrm{pH}$ and other physical characteristics. Although due to economical concern, formulation made by using $10 \%$ acacia was chosen as an optimum formulation.

Release profile between Corlan ${ }^{\circledR}$ and FI (acacia 10\%) was comparable. There was no significant difference $(p<0.05)$ between release of reference and FI tablets according to one-way ANOVA. Release pattern was fitted

Table 2: Physicochemical properties of mucoadhesive buccal tablets of Hydrocortisone Sodium Succinate and Corlan.

\begin{tabular}{|c|c|c|c|c|c|c|c|c|c|}
\hline Formulations & $\begin{array}{c}\text { Weight average } \\
\text { (mg) }\end{array}$ & Friability & $\begin{array}{c}\text { DT } \\
\text { (min.) }\end{array}$ & $\begin{array}{c}\text { Thickness } \\
\text { (mm) }\end{array}$ & $\begin{array}{c}\text { Hardness } \\
\left(\mathrm{kg} / \mathrm{cm}^{2}\right)\end{array}$ & Assay & $\begin{array}{c}\text { Surface } \\
\mathrm{pH}\end{array}$ & $\begin{array}{l}\text { Content } \\
\text { uniformity }\end{array}$ & $\begin{array}{c}\mathrm{D} \\
(\mathrm{mm})\end{array}$ \\
\hline F I & 80.0 & $<1 \%$ & $10^{\prime}, 58^{\prime \prime}$ & 1.6 & 7.1 & 99.0 & 6.76 & $96.0 \mathrm{AV}<15$ & 6.0 \\
\hline FII & 80.0 & $<1 \%$ & $12^{\prime}, 20^{\prime \prime}$ & 1.6 & 7.6 & 100.0 & 6.77 & $97.0 \mathrm{AV}<15$ & 6.0 \\
\hline FIII & 80.0 & $<1 \%$ & $13^{\prime}, 45^{\prime \prime}$ & 1.6 & 7.8 & 98.5 & 7.65 & $97.0 \mathrm{AV}<15$ & 6.0 \\
\hline Corlan & 75.0 & $<1 \%$ & $14^{\prime}$ & 1.2 & 7.1 & 99.0 & 6.7 & $97.0 \mathrm{AV}<15$ & 5.5 \\
\hline
\end{tabular}

$\mathrm{DT}=$ Disintegration time, $\mathrm{D}=$ Diameter, $\mathrm{AV}=$ Average
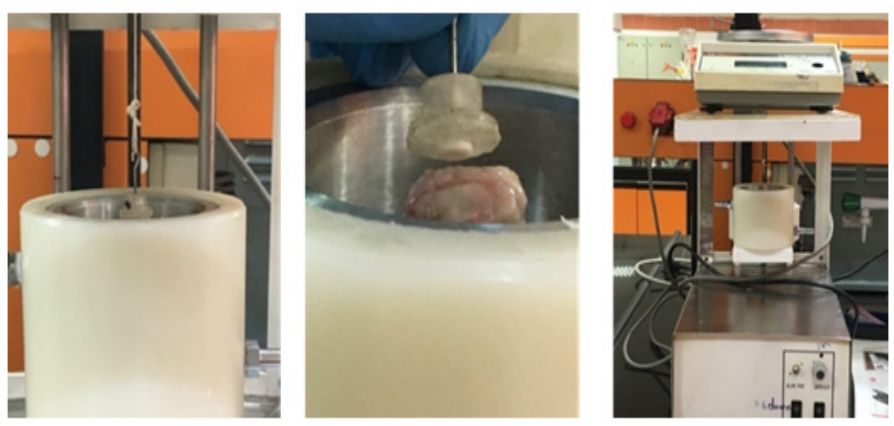

Figure 2: Mucoadhesive strength testing apparatus.

Table 3: Mucoadhesive strength of buccal tablets of Hydrocortisone sodium succinate and Corlan.

\begin{tabular}{ccc}
\hline Formulation code & $\begin{array}{c}\text { Mucoadhesive } \\
\text { strength }(\mathrm{g})\end{array}$ & $\begin{array}{c}\text { Mucoadhesive force } \\
(\mathrm{N})\end{array}$ \\
\hline FI & $3.71 \pm 0.2$ & $0.36 \pm 0.02$ \\
FII & $4.27 \pm 0.2$ & $0.42 \pm 0.02$ \\
FIII & $6.19 \pm 0.4$ & $0.61 \pm 0.04$ \\
$\begin{array}{c}\text { Reference tablet } \\
\text { (Corlan) }\end{array}$ & $2.49 \pm 0.2$ & $0.24 \pm 0.02$ \\
\hline
\end{tabular}

(Mean of $3 \pm$ SD) to a number of mathematical kinetic models of release. According to results the best model for our optimized formulation is the HixsonCrowell model (Table 4). It seems that our release mechanism relies in dissolution from a continually changing surface of tablet during the process. ${ }^{25}$ Results were different for Corlan release pattern and degree of correlation was low that was indicating a poor relationship with predicted model (Weibull, RSQ: 0.883, MPE: 5.30).

In-vitro bio-equivalency was performed on the basis of a simple and independent mathematical model using difference factor (f1) and

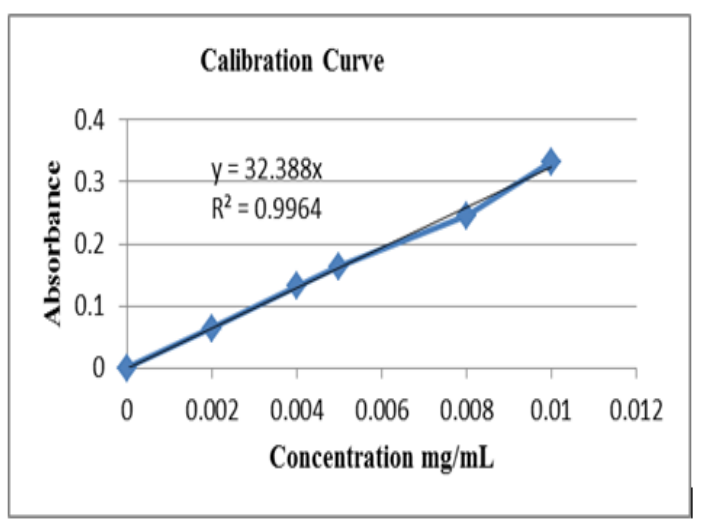

Figure 3: Hydrocortisone sodium succinate calibration curve at $248 \mathrm{~nm}$. 


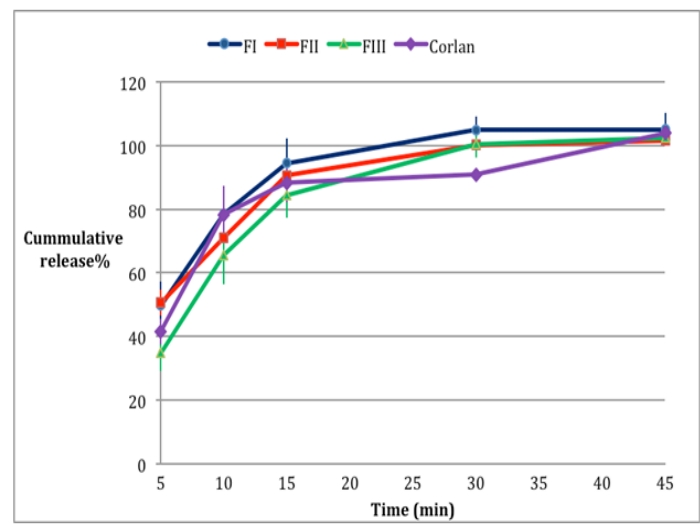

Figure 4: Comparison of release profile for Fl, Fll, FIII and Corlan. (Mean of $3 \pm \mathrm{SD}$ ).

Table 4: Parameters of release kinetic models for FI formulation.

\begin{tabular}{ccccc}
\hline Model & MPE & RSQ & Slope & Intercept \\
\hline Zero order & 13.87 & 0.824 & 0.014 & 0.445 \\
First order & 44.10 & 0.859 & -0.203 & 1.320 \\
Higuchi & 9.41 & 0.912 & 0.134 & 0.165 \\
Hixson-Crowell & 4.08 & 0.984 & 0.019 & 0.068 \\
Square root of mass & 7.09 & 0.973 & 0.018 & 0.182 \\
Weibull & 4.53 & 0.938 & 1.246 & -2.889 \\
Linear probability & 3.74 & 0.954 & 0.093 & -0.774 \\
Log probability & 12.80 & 0.839 & 1.639 & -3.350 \\
$\begin{array}{c}\text { Reciprocal powered } \\
\text { mass }\end{array}$ & 20.38 & 0.729 & -3.783 & 7.868 \\
\hline
\end{tabular}

similarity factor (f2) obtained from release profile. Similarity factor is approved by FDA for immediate-release solid oral medicines. ${ }^{28}$ Similarity and difference factors were calculated by using number of time points (n), dissolved amount of reference tablet at time $t\left(\mathrm{R}_{\mathrm{t}}\right)$ and dissolved amount of test tablet at time $\mathrm{t}\left(\mathrm{T}_{\mathrm{t}}\right)$. According to the literature two formulations are considered bioequivalent if $\mathrm{f} 1$ is between 0 and 15 and $\mathrm{f} 2$ is in the range of 50 to $100 .{ }^{29}$ For our FI formulation in comparison to the reference mucoadhesive tablets, $\mathrm{f} 1$ was 9.24 and $\mathrm{f} 2$ was equal to 50.95 (Table 5). It is concluded that FI is similar to Corlan and may be used instead.

\section{DISCUSSION}

As mentioned before, buccal adhesive systems offer some advantages in terms of accessibility, ease of administration and withdrawal, keeping in situ and high patient compliance. Adhesion of these drug delivery devices to mucosal membrane leads to an increased drug concentration at the absorption site and therefore improves availability of delivered drugs. Adhesiveness increases the contact time over the lesion mucosa. Shortening of treatment time is therefore another advantage of buccal delivery for mouth disorders. In addition to increase in drug delivery to the action site, these systems reduce side effects compared to the systemic administration. It is concluded that buccal adhesive dosage forms are excellent dosage forms for mouth local disorders. ${ }^{30}$

Several classes of polymers are used as mucoadhesive compounds. Natural mucoadhesive polymers are getting more attention recently because of low cost and toxicity, biodegradability and safety over synthetic mucoadhesive compounds. Chitosan, alginate and cellulose
Table 5: In-vitro bioequivalence test result for FI and Corlan tablets.

\begin{tabular}{cccccc}
\hline Time (min) & \multicolumn{2}{c}{ Dissolution } & $\mathrm{T}_{\mathrm{t}}-\mathrm{R}_{\mathrm{t}}$ & $\mathrm{T}_{\mathrm{t}}-\mathrm{R}_{\mathrm{t}}$ & $\left(\mathrm{T}_{\mathrm{t}}-\mathrm{R}_{\mathrm{t}}\right)^{2}$ \\
& $\mathrm{Tt}$ & $\mathrm{Rt}$ & & & \\
\hline 0 & 0 & 0 & 0.00 & 0.00 & 0.00 \\
5 & 37.95 & 41.55 & -3.60 & 3.60 & 12.96 \\
10 & 60.32 & 78.30 & -18.02 & 18.02 & 324.7 \\
15 & 78.30 & 88.18 & -9.88 & 9.88 & 97.61 \\
30 & 92.74 & 90.83 & 1.91 & 1.91 & 3.65 \\
45 & 100.09 & 103.87 & -3.78 & 3.78 & 14.29 \\
Sum & 369.40 & 402.73 & -37.33 & 41.15 & 453.23 \\
\multicolumn{5}{c}{$\mathrm{f} 2=50 * \log \left\{\left[1+1 . n \sum(\mathrm{Rt}-\mathrm{Tt})^{2}\right]^{-0.5 *} 100\right\}=$} & 50.95 \\
\multicolumn{5}{c}{$\mathrm{f} 1=\sum(|\mathrm{Rt}-\mathrm{Tt}|) .\left(\sum \mathrm{Rt}\right)^{*} 100=$} \\
\hline \multicolumn{5}{c}{}
\end{tabular}

derivatives are among these polymers. ${ }^{31}$ Acacia may be considered as one of natural adhesives with high mucoadhesion strength. ${ }^{30}$ Furthermore as we had decided to compare our formulated buccal tablet with Corlan in which acacia was used for mucoadhesion, therefore acacia was chosen for the formulation.

Using acacia, proper mucoadhesion was achieved. The mucoadhesive strength with the lowest concentration of acacia was comparable to Corlan tablets. These buccal tablets showed a fast in-vitro dissolution profile that serves to release a high amount of drug in a short time to promote healing of the aphthous lesions. The entire drug formulated in tablets was released successfully. Results of in-vitro tests were quite similar and comparable to Corlan tablets showing a promising formulation. Increase in acacia concentration did not produce a significant change $(p<0.05)$ in the release profile. Although in many studies concentration of mucoadhesive compound caused a significant decrease in release rate. ${ }^{21}$ It may be related to the fact that formulated hydrocortisone sodium succinate tablet has a fast dissolving property.

Model of release kinetic was also determined according to the formula of different release models and found out to be obeying the Hixson-Crowell best. This is a release pattern on the basis of dissolution of particles from progressively changing surface of a matrix.

It has been repeatedly reported that similar pharmaceutical products show different effects in-vivo. Comparison of dissolution profile may be considered a fairly precise in-vitro method. A simple method of calculation that is independent from type of formulation was employed for this purpose. Difference factor (f1) shows the percentage of difference between formulated and reference release profile at each time point. Similarity factor (f2) measures the similarity between releases of formulations that has been adopted by FDA for in-vitro bioequivalence studies. According to f1 and $\mathrm{f} 2$ factors, our formulated buccal tablet is expected to be similar to Corlan in-vivo.

\section{CONCLUSION}

Mucoadhesive buccal systems are offering superior therapy for localized oral cavity disorders like aphthous lesions. In this study a buccal tablet with mucoadhesive property was successfully formulated and compared to a reference tablet in-vitro. Mucoadhesive strength of our tablet was similar to the reference tablet. Released amounts after 45 min were comparable. Release pattern were fit with the Hixson-Crowell model. Invitro bioequivalence study (similarity and dissimilarity factor) showed that our formulated buccal tablet of hydrocortisone may be considered bioequivalent to the reference tablet meaning that it may be used interchangeably. 
The future expectation of buccal adhesive drug delivery may be accounted for the efficient delivery of proteins and peptide compounds. Study on the new natural or synthetic adhesive polymers will result in growing interest for this type of formulation for management of painful ulcerative lesions inside the mouth in chemotherapy and immunocompromised patients. Studies should also be directed to develop more validated and standard in-vitro methods in order to provide the accurate assessment of buccal adhesive systems to promote local and systemic delivery via oral mucosa.

\section{ACKNOWLEDGEMENT}

Thanks to Dr. Seyed Esmaeil Sadat Ebrahimi, Head of the School of Pharmacy, International Campus of Tehran University of Medical Sciences, for his collaboration and support for conducting the study. The Authors would also like to acknowledge the technical assistance received from Eng. Mohammad Ali Kochanani.

\section{CONFLICT OF INTEREST}

The authors declare no conflict of interest.

\section{ABBREVIATIONS}

AV: Average; D: Diameter; f1: Difference Factor; DT: Disintegration Time; g: Gram; MPE: Mean Percentage of Error; $\mu \mathrm{m}$ : Micrometer; mg: Milligram; ml: Milliliter; mm: Millimeter; min: Minute (s); nm: Nanometer; N: Newton; n: Number; Rt: Reference at the time t; RSQ: Residual Sum of squares; rpm: Rounds Per Minute; f2: Similarity Factor; $\Sigma$ : Sum; Tt: Test at the time t; USP: United States Pharmacopoeia.

\section{REFERENCES}

1. Yakubov GE, Gibbins H, Proctor GB, et al. Oral Mucosa: Physiological and Physicochemical Aspects. Mucoadhesive Materials and Drug Delivery Systems. 2014;1-38.

2. Ceschel GC, Maffei $P$, Borgia SL, Ronchi C. Design and Evaluation of Buccal Adhesive Hydrocortisone Acetate (HCA) Tablets. Drug Deliv. 2001;8(3):161-71. https://doi.org/10.1080/107175401316906937

3. Oral mucosal drug delivery and therapy.: Springer. 2015.

4. Laffleur F, Küppers P. Adhesive alginate for buccal delivery in aphthous stomatitis. Carbohydr Res. 2019;477:51-7.

5. Muñoz-Corcuera M, Esparza-Gómez G, González-Moles MA, et al. Oral ulcers: Clinical aspects: A tool for dermatologists. Part I. Acute ulcers. Clin Exp Dermatol. 2009;34(3):289-94. .https://onlinelibrary.wiley.com/doi/abs/10.1111/ j.1365-2230.2009.03220.x

6. Chinna RP, Chaitanya KSC, Madhusudan RY. A review on bioadhesive buccal drug delivery systems: current status of formulation and evaluation methods. Daru. 2011;19(6):385-403. https://www.ncbi.nlm.nih.gov/pubmed/23008684

7. Quijano D, Rodríguez M. Corticoides tópicos en la estomatitis aftosa recurrente. Revisión sistemática. Acta Otorrinolaringol Esp. 2008;59(6):298-307. http:// www.sciencedirect.com/science/article/pii/S0001651908733144

8. Russo E, Selmin F, Baldassari S, et al. A focus on mucoadhesive polymers and their application in buccal dosage forms. J Drug Deliv Sci Technol. 2016;32:11325. http://www.sciencedirect.com/science/article/pii/S1773224715001112

9. Khalid I, Ahmad M, Minhas MU, et al. Formulation and in vitro evaluation of mucoadhesive controlled release matrix tablets of flurbiprofen using response surface methodology. Braz J Pharm Sci. 2014;50:493-504. http://www.scielo.br/
scielo.php?script=sci_arttext\&pid=S1984-82502014000300493\&nrm=iso

10. Nafee NA, Ismail FA, Boraie NA, et al. Mucoadhesive Delivery Systems. II. Formulation and in-vitro/in-vivo Evaluation of Buccal Mucoadhesive Tablets Containing Water-Soluble Drugs. Drug Dev Ind Pharm. 2004;30(9):995-1004. https://doi.org/10.1081/DDC-200037226

11. Salamat-Miller N, Chittchang M, Johnston TP. The use of mucoadhesive polymers in buccal drug delivery. Adv Drug deliver Rev. 2005;57(11):1666-91

12. Shanker G, Kumar CK, Gonugunta CSR, et al. Formulation and evaluation of bioadhesive buccal drug delivery of tizanidine hydrochloride tablets. AAPS PharmSciTech. 2009;10(2):530-9. https://www.ncbi.nlm.nih.gov/ pubmed/19424804

13. Velmurugan S, Deepika B, Nagaraju K, et al. Formulation and in-vitro Evaluation of Buccal Tablets of Piroxicam. Int J Pharmtech Res. 2010;2(3):1958-68.

14. Guo YG, Pratap SA. Emerging strategies for enhancing buccal and sublingual administration of nutraceuticals and pharamaceuticals. J Drug Deliv Sci Technol. 2019;52:440-51. http://www.sciencedirect.om/science/article/pii/ S1773224719300401

15. Hao J, Heng PWS. Buccal Delivery Systems. Drug Dev Ind Pharm. 2003;29(8):821-32. https://doi.org/10.1081/DDC-120024178

16. Rowe RC, Sheskey P, Quinn M. Handbook of pharmaceutical excipients: Libros Digitales-Pharmaceutical Press. 2009

17. Pharmaceutical Dosage Forms: Tablets. CRC Press. 2008.

18. Bagul U, Gujar K, Dhat S, et al. In vitro study of mucoadhesive strength of polymers for mucoadhesive drug delivery systems. Int J Curr Pharm Res. 2009:1:42-6.

19. Patil P, Kulkarni SV, Someshwara Rao B, et al. Formulation and in vitro evaluation of mucoadhesive tablets of ofloxacin using natural gums. Int J Curr Pharm Res. 2011;3(2):93-8.

20. Mahalaxmi D, Senthil A, Prasad $V$, et al. Formulation and evaluation of mucoadhesive buccal tablets of glipizide. Int J Biopharm. 2010;1(2):100-7. https:// pdfs.semanticscholar.org/87bc/b0e112260b8595605b5fe5b6f012ba46f095.pdf

21. Moghimipour E, Aghel N, Adelpour A. Formulation and Characterization of Oral Mucoadhesive Chlorhexidine Tablets using Cordia myxa Mucilage. Jundishapur J Nat Pharm Prod. 2012;7(4):129-33. https://www.ncbi.nlm.nih.gov/pmc/ articles/PMC3941876/pdf/jjnpp-07-129.pdf

22. Dinesh BG, Reddy BV, Sekhar MH, et al. Design and Evaluation of tablets of Nifedipine. JPSI. 2012.

23. Mehravaran N, Moghimi $H$, Mortazavi SA. The Influence of various mucoadhesive polymers on in vitro performance of the resulting artificial saliva pump spray formulations. IJPR. 2010;3-13. http://journals.sbmu.ac.ir/ testtesttest/article/view/1049

24. Carvalho FC, Bruschi ML, Evangelista RC, et al. Mucoadhesive drug delivery systems. Braz J Pharm Sci. 2010;46(1):1-17.https://onlinelibrary.wiley.com/doi/ book/10.1002/9781118794203

25. Mircioiu C, Voicu V, Anuta V, et al. Mathematical Modeling of Release Kinetics from Supramolecular Drug Delivery Systems. Pharmaceutics. 2019;11(3):140. https://www.ncbi.nlm.nih.gov/pmc/articles/PMC6471682/

26. Zakeri-Milani $P$, Nayyeri-Maleki $P, G$ hanbarzadeh $S$, et al. In-vitro bioequivalence study of 8 brands of metformin tablets in Iran market. J Appl Pharm. 2012;2(8):194. https://pdfs.semanticscholar.org/1696/2fe3b29743b586176877f acaf92df15a08f3.pdf

27. Jelvehgari M, Rashidi MR, Samadi H. Mucoadhesive and Drug Release Properties of Benzocaine Gel. IJPR. 2006;2(4):185-94.http://www.ijps.ir/article_ 1945_988553a735143686dd84fc8a81b76b51.pdf

28. FDA Guidance for Industry. Dissolution testing of immediate release solid dosage forms. 1997.

29. Nayak AK, Pal DJIJOPS. Research. Comparative in vitro bioequivalence analysis of some ciprofloxacin HCl generic tablets. 2010;1(8):51

30. Boateng J, Ayensu I, Pawar HC. Mucoadhesive materials and drug delivery systems.: John Wiley and Sons. 2014;233-54

31. Katsarov $P$, Pilicheva $B$, Uzunova $Y$, et al. Chemical cross-linking: A feasible approach to prolong doxylamine/pyridoxine release from spray-dried chitosan microspheres. Eur J Pharm Sci. 2018;123:387-94. https://www.sciencedirect. com/science/article/pii/S0928098718303634?via\%3Dihub 